\title{
Context-based microblog browsing for mobile users
}

\author{
Jonghyun Han ${ }^{\mathrm{a}}$, Xing Xie ${ }^{\mathrm{b},{ }^{*}}$ and Woontack Woo ${ }^{\mathrm{c}}$

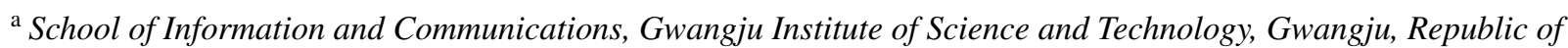 \\ Korea \\ E-mail: jhan@gist.ac.kr \\ b Microsoft Research Asia, Beijing, China \\ E-mail:xing.xie@microsoft.com \\ ${ }^{\mathrm{c}}$ KAIST U-VR Lab., Daejon, Republic of Korea \\ E-mail:wwoo@kaist.ac.kr
}

\begin{abstract}
Mobile microblog browsing is inconvenient due to the limitations of mobile devices. Therefore, it is important to effectively retrieve relevant and timely microblog content that caters to the information need of mobile users. In this paper, we first present the findings from a large-scale study on the relationship between microblog content and user context. Then, we show a system that detects local microblog topics, estimates user interests and selects user-preferred topics. The system employs user context to detect microblog topics and post-processes the topics for finding user-preferred content. We exploit time, location, browsing history, social relationship and activity as user context. The effectiveness of our approach is evaluated against several baseline algorithms for investigating the impact of user context on the relevance of retrieved topics. According to our experimental results, the approach enhances the relevance of topics by $24 \%$, compared to the baseline approaches. Thus, we expect that the proposed approach is helpful in advancing mobile information retrieval.
\end{abstract}

Keywords: Mobile context-awareness, microblog analysis, mobile information browsing, microblog topic retrieval, mobile content recommendation

\section{Introduction}

Microblog services have been one of the most popular services on mobile devices. Because of the tremendous popularity of them, there has been an explosive increase in the quantities of microblogs. Many users need to browse microblogs on mobile phones for getting timely information on the site. Due to the improvements in the performance of mobile phones, the microblog services are usually combined with intelligent services, such as location-based services. However, the limitations of mobile phones - e.g., small display and low computational power - still present difficulties in the retrieval and visualization of large quantities of microblogs. Thus, it is important to effectively

\footnotetext{
*Corresponding author
}

retrieve microblog content related to the information needs of mobile users.

Recently, many studies have dealt with understanding the information needs of mobile users. One research field related to understanding mobile information need is mobile context-aware computing. Although mobile applications have limitations compared to desktop applications, it is easier to take advantage of a user's contextual information because mobile phones are very personal and have built-in sensors. Contextual information allows websites and applications to provide user-centric content $[1,22,24]$. Mobile context-awareness has been applied to mobile information retrieval $[4,10,20]$. There are also studies related to microblog content retrieval or recommendation $[5,15,17]$. Those studies mainly focus on extracting information from microblogs, instead of under- 


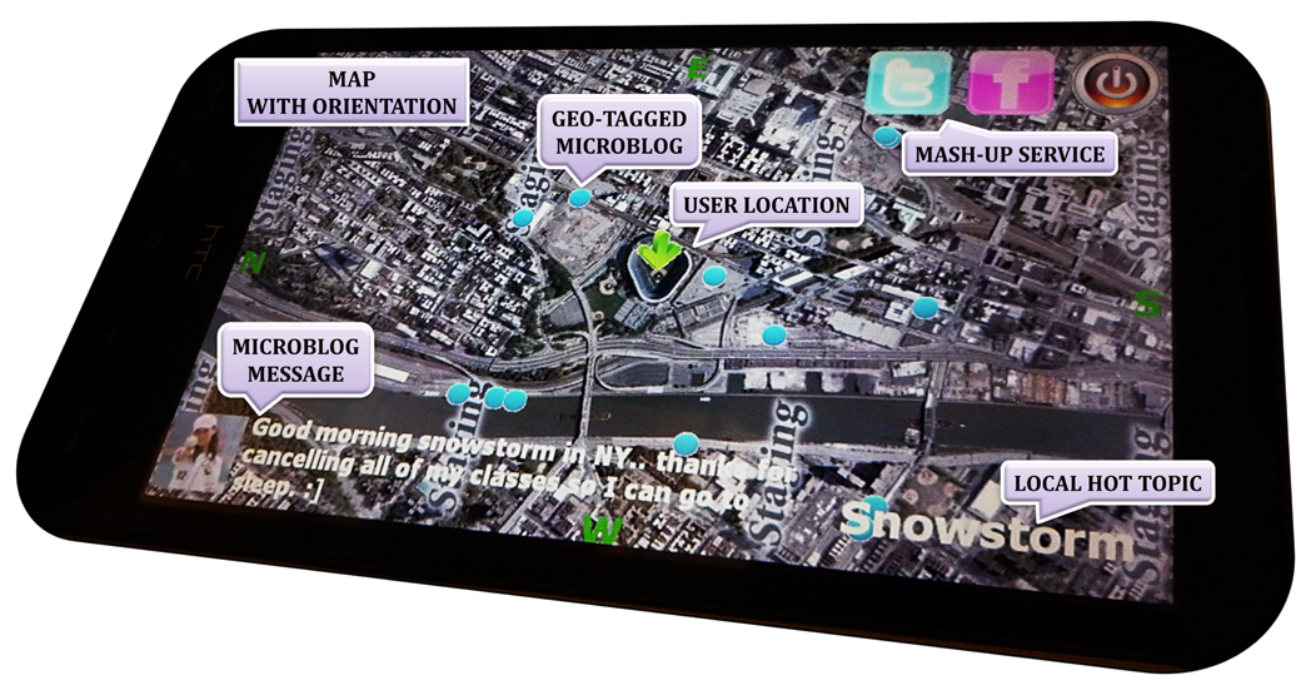

Fig. 1. The user interface of Context-aware Mobile Microblog Browser.

standing user information needs. In order to overcome difficulties in the retrieval of user-preferred microblogs on a mobile device, it is necessary to consider user context, such as location and social network.

In this paper, we studied the problem of finding content relevant to the information needs of mobile users. We examined the impact of user context on microblog usage, and presented a system that provided users with user-preferred microblog topics (see Fig. 1). We presented the findings of a large-scale study on the relationship between microblogs and user context expressed on a microblog service. First, we examined the impact of context on the frequency of terms used in microblogs. Second, we observed the distribution of microblogs across categories when activity information is given. Finally, we determined important factors for finding user-preferred microblog content.

We proposed an approach to effectively retrieve microblog topics relevant to a mobile user's needs. To overcome difficulties in visualizing large quantities of microblogs on a mobile device, we summarized the microblogs as topics that describe recent emerging issues. Our approach involved detecting local microblog topics, defined as topics mentioned frequently over a period of time by local users; estimating user interests from user context such as user history, social relationship, and user activity; and post-processing the top results in accordance with the estimated user interests for selecting user-preferred microblog topics. Using our observations concerning the impact of user context on microblog usage, we were able to develop an ap- proach that personalized microblog topics with greater relevance.

We evaluated our approach to the problem and tried to investigate the impact of user context on the relevance of retrieved microblog topics. According to our evaluation, our proposed approach enhanced the relevance of microblog topics to a mobile user. In general, the best algorithm was to post-process local microblog topics with user interests. When we employed user activities for user interests, it achieved a topic relevance of 0.748 and normalized discounted cumulative gain (nDCG) of 0.768 . The proposed approach enhanced the relevance of topics by $24 \%$, compared to baseline approaches. Our evaluation indicated that the proposed approach is effective in advancing the relevance of microblog topic retrieval on a mobile device.

The organization of the rest of this paper is as follows: Section 2 is devoted to the background of our approach. We examine the relationship between microblogs and contextual information in Section 3. Our proposed approach that retrieves local microblog topics from microblogs is described in Section 4. In Section 5, we show the evaluation of local microblog topic detection. Conclusion and future works are presented in Section 6.

\section{Background}

\subsection{Related works}

One of the research areas related to our approach is context-awareness in a ubiquitous computing envi- 
ronment. A number of studies have been conducted to exploit mobile context for developing user-centered applications [1,14,21,22,24]. Abowd et al. provided a personal guide to exploiting mobile user context such as knowledge of the user's current location and a history of past locations [1]. Sun and May described the aspects of spatial context for the design of personally relevant mobile services through user studies [21], and $\mathrm{Xu}$ et al. showed how a mobile user's intention is affected by mobile context [24]. In [21], user interests and preferences are influenced by location. Tamminen et al. examined how context-aware computing takes effect in mobile activity with characteristics of mobile context [22]. Lemmelä et al. presented the design process for mobile multimodal applications by exploiting varied context of daily life [14]. These studies conducted user studies with a few participants to observe the importance of mobile context.

Context-awareness can be applied to mobile search to understand the information needs of mobile users. Many technologies have been presented to help discover mobile information needs $[8,9,13,26]$. Kamvar et al. [13] and Yi et al. [26] presented the characteristics of mobile search queries. They examined the categories of mobile queries through large-scale data analysis. Church et al. proposed an approach for supporting context-sensitive mobile search [8] and showed how to understand mobile information needs through user studies [9]. They stated that context, such as location and time, are important factors to understand the intent of mobile users. From these works, we are able to understand which information is needed by mobile users. We need to consider the changes of information needs when there are events around users or users are in specific situations - e.g., working or exercising.

Another area of research related to this paper is information retrieval, especially topic detection. Many methods have been introduced to evaluate the significance of a keyword in documents $[3,6,16,25]$; they involve giving weight to important words of a corpus. The most well-known way to give weight to terms is Term Frequency - Inverse Document Frequency (TFIDF) [19]. It evaluates the significance of a term in a document based on the number of times it appears in the document and the frequency of the term in the corpus. Chen et al. used a term-weighting scheme with aging theory [6] and Brants et al. used an incremental TF-IDF model based on document-specific averages [3]. In [18], Sekiguchi et al. detected topic words from blog documents. They defined a topic as frequently used words and proposed an approach using the blog- ger's interest vector. Most of literature has focused on the significance of terms in a document. In this paper, we propose an approach for detecting local and recent rising topics and estimating a user's interest from short microblog messages.

In recent years, researchers have investigated mobile context-aware information retrieval by exploiting mobile context-awareness and information retrieval technologies $[4,23]$. According to Brown and Jones, in order to improve the efficiency and effectiveness of information retrieval, it is necessary to apply a contextaware retrieval approach where display space is limited, particularly [4]. The Context-Aware Browser for mobile devices provides relevant Web documents based on inferred user current context [10]. In [20], in order to recommend music for mobile users, $\mathrm{Su}$ et al. took advantage of context information mining with collaborative filtering. Church et al. presented an approach to discover information for mobile users [7]. They showed that social context was a key factor in improving the information discovery experience of mobile users.

Although mobile context has been a topic of studies across many research areas, the observations of the previous works have been focused on information retrieval using location $[9,21]$. In this paper, we would like to observe the importance of various mobile contextual factors for understanding the information needs of mobile users. First, we investigate temporal, spatial, and social properties of mobile context based on largescale microblog data sets. The investigation of mobile context makes us understand which factors are important for providing user-preferred content. We also analyze the changes of information needs when contextual situations are given. Then, we describe the use of these analysis results in a microblog topic detection and selection model.

\subsection{Terminology}

Microblog In this paper, we use microblog to mean a source of information in which a mobile user has interest. It is a kind of communication tool used within a social network or publicly. The main difference between a blog and a microblog is the length of the text messages. Microblog services provide a brief text message describing a user's current status or opinion. Because a microblog's message is short, users generally update their microblogs frequently and the microblogs reflect real-time issues. In addition, microblog services usually include a social networking component. Users can 
follow other users' microblogs when they want to read them. Links between users are not always symmetric, so the microblog can generate directed networks based on a user's microblog interests. In this paper, we assume that a network built using the user's following information is a kind of social network.

Microblog topic A topic is used either as a keyword representing a document or as an area of interest. In microblogs, a topic is a term which is mentioned in a microblog over a period of time. In this paper, we define a microblog topic as a topic which is frequently mentioned in microblogs. A local microblog topic means a microblog topic mentioned frequently by local users. We consider unigram terms only as topics, and consider recently emerging issues instead of constantly mentioned issues.

Context We employ user context to retrieve local microblogs and estimate user interests. We use temporal, spatial, personal information, and user activities as user context. As user spatial context, we use not only sensory data obtained by mobile phone sensors - for instance, latitude and longitude, but also locations described in user profiles of a microblog service. The spatial context is used to narrow down the search range of microblogs to the boundary area where a user is located. User personal information, including blogging history and social relationships, is used to obtain user interests. Since a microblog service is a kind of social media, we consider social relationship as an important property of microblogs. By exploiting a G-sensor, a compass, Global Positioning System (GPS) trajectories, and time, we can infer user activities - for example, 'before go to work from home' or 'walking down the street in the afternoon'. Examples of context used in this paper are shown in Table 1.

\section{Relationship between microblog and context}

We first analyze how context affects microblogs in order to browse microblogs by exploiting contextual information. In this analysis, we present the findings of a large-scale study on the relationship between microblogs and user context.

\subsection{Data set}

In this examination, we use Twitter $^{1}$, a well-known microblog service, as microblog data. Twitter enables

${ }^{1}$ Twitter, http://www.twitter.com
Table 1

Examples of user context used in our system

\begin{tabular}{ll}
\hline Type & \multicolumn{1}{c}{ Examples } \\
\hline Temporal & $\begin{array}{l}\text { morning/afternoon/night, lunch/dinner, } \\
\text { working day, weekend, ... } \\
\text { latitude, longitude, orientation } \\
\text { home, work, school, shop, restaurant, ... } \\
\text { Spatial }\end{array}$ \\
& United States, New York, California, ... \\
Personal & social relationship (following, follower) \\
Activity & working, studying, shopping, exercising, ...
\end{tabular}

users to post and read short messages that describe their status. It also provides a feature of social networking. A user is able to follow other users when he has an interest in microblogs posted by others. We regard only following information as social relationships. A user is also able to input the user's profiles, including location, language, and a brief description.

We observe the impact of spatial context, such as user location when a user uses microblogs. To extract user location, we use the location information described in the service's profile. The profile information is not mandatory, so users can input their profile freely. Although some of the location information may be incorrect, we assume that most of them give us the user's location. Because the location field of the profile is optional, we assume that addresses entered by users are almost correct. We use the cities of the addresses and convert latitude and longitude into names of cities by reverse-geocoding.

We gather and use microblogs posted by users located in the United States (US) from December 13th, 2009, to January 31st, 2010. The US microblogs are used as a global data set. The number of global microblogs is approximately 55 million, and of these 55 million microblogs with locations, 4.5, 1.9, and 3.2 million microblogs are posted by New York (NY), Chicago $(\mathrm{CH})$, and California $(\mathrm{CA})$ users, respectively. In this paper, we use these locations and microblogs to examine the relationship between microblogs and context. To avoid any language problem, we only use microblogs posted in English.

\subsection{Analysis approach}

In this paper, we compare term frequency (TF) of two sets of microblogs in order to observe the impact of context on microblogs. TF is computed by the occurrence count of a term in a document. However, 
in contrast with a document, a microblog is too short to compute term frequency. In order to avoid biased TF values in a microblog, we use a set of microblogs posted during a specified duration as a document. We use TF with Cosine similarity measurement to compute similarity between microblog sets.

We divide microblogs data into six sets. One of the sets is a test microblog set, which was posted by randomly selected users within a given duration. In this analysis, we use one day as a duration parameter. Another set is a baseline collection of all of microblogs except microblogs belonging to the test set. In other words, the baseline set does not exploit any contextual information. And the other four sets are collections of microblogs containing each contextual information respectively; location, history, social relationships, and local social relationships.

For a set from location context, we gather microblogs posted by users who are located in the same cities with the test set users. For instance, we retrieve $\mathrm{NY}, \mathrm{CH}$, and CA microblogs from the baseline set when we analyze the test set posted by these locations. For the impact of user history, we generate a history set that contains previous microblogs posted by the test set users. A set for social relationships consists of microblogs posted by friends of the test set users. The last set is a collection of microblogs posted by local friends of the test set users. In other words, the set consists of microblogs posted by friends who live in same cities as the test set users.

We compute the TF of each set of microblogs in order to compute the similarity between a test set and sets of comparison groups. To enhance the accuracy of the similarity, we remove unimportant words using a stopword elimination task. We also use a stemming task to solve problems caused by tenses and plural forms. Next, the top 100 most occurring terms in descending order are selected for similarity measurement. Then, we measure closeness between the test set and each of the other sets. The similarity is computed using Cosine similarity measurement [2], as defined in Eq. (1). In Eq. (1), $\mathrm{TF}_{\mathbf{A}}(t)$ represents the term frequency of a term $t$ in a microblog set $\mathbf{A}$. Then, we find the impact of each contextual information through the similarity.

$\operatorname{TF} \operatorname{sim}(\mathbf{A}, \mathbf{B})=\frac{\sum_{t \in\{\mathbf{A} \cap \mathbf{B}\}} \mathrm{TF}_{\mathbf{A}}(t) \cdot \mathrm{TF}_{\mathbf{B}}(t)}{\sqrt{\sum_{t \in \mathbf{A}} \mathrm{TF}_{\mathbf{A}}^{2}(t)} \sqrt{\sum_{t \in \mathbf{B}} \mathrm{TF}_{\mathbf{B}}^{2}(t)}}$
In addition, to examine how a user activity affects usage of microblogs, we check which kinds of categories are interesting when a user activity is given. Because a microblog does not contain a tag for activity information, it is difficult to retrieve microblogs posted in a given situation. Thus, we retrieve and exploit microblogs that mention specific terms describing an activity or situation - for instance, 'shopping', 'working' or 'in the school'. From the retrieved microblogs, we find other terms frequently mentioned with the terms that describe an activity or situation. Then we categorize the terms (described in detail in Section 4.3.2), after which we find a pattern of microblog usage when the activity terms are mentioned.

\subsection{Analysis results}

Figure 2 shows the TF similarity results between a test microblog set and the other microblog sets. In general, as Fig. 2 shows, TF similarities of microblog sets using contextual information are higher than a similarity of a baseline set that does not exploit any context. Even though the absolute values of the similarities are small due to a broad spread of term distribution in the microblogs, we can see the relative differences between microblog sets. The set collected with user history context has the highest similarity value $(0.231)$, and the baseline set has the lowest similarity (0.133), on average. The similarities of the other sets collected with context are higher than that of the baseline set. Therefore, in accordance with this analysis, we find that microblog usage is affected by user context.

TF similarity is partially affected by temporal context, such as date. As shown in Fig. 2, the similarities of the baseline and the spatial microblog set exceed 0.2 on special days, such as Christmas and New Year's Day, and are much higher than those of the other days. Because many microblogs of the day deal with greeting messages, the impact of temporal context is increased. On January 15th, 2010, 'Haiti' and 'donate' were the most frequently mentioned terms because of the 2010 Haiti earthquake. On the day, the similarity of the global set (0.143) and that of the spatial set (0.161) are higher than other days. In addition, day of the week influences term frequencies in microblogs. For example, 'working' is mentioned frequently on weekdays, but 'church' is mentioned frequently on weekends. So, temporal context influences a pattern of microblog usage.

Spatial context also affects the TF of microblogs. TF similarity of a spatial microblog set is always higher 


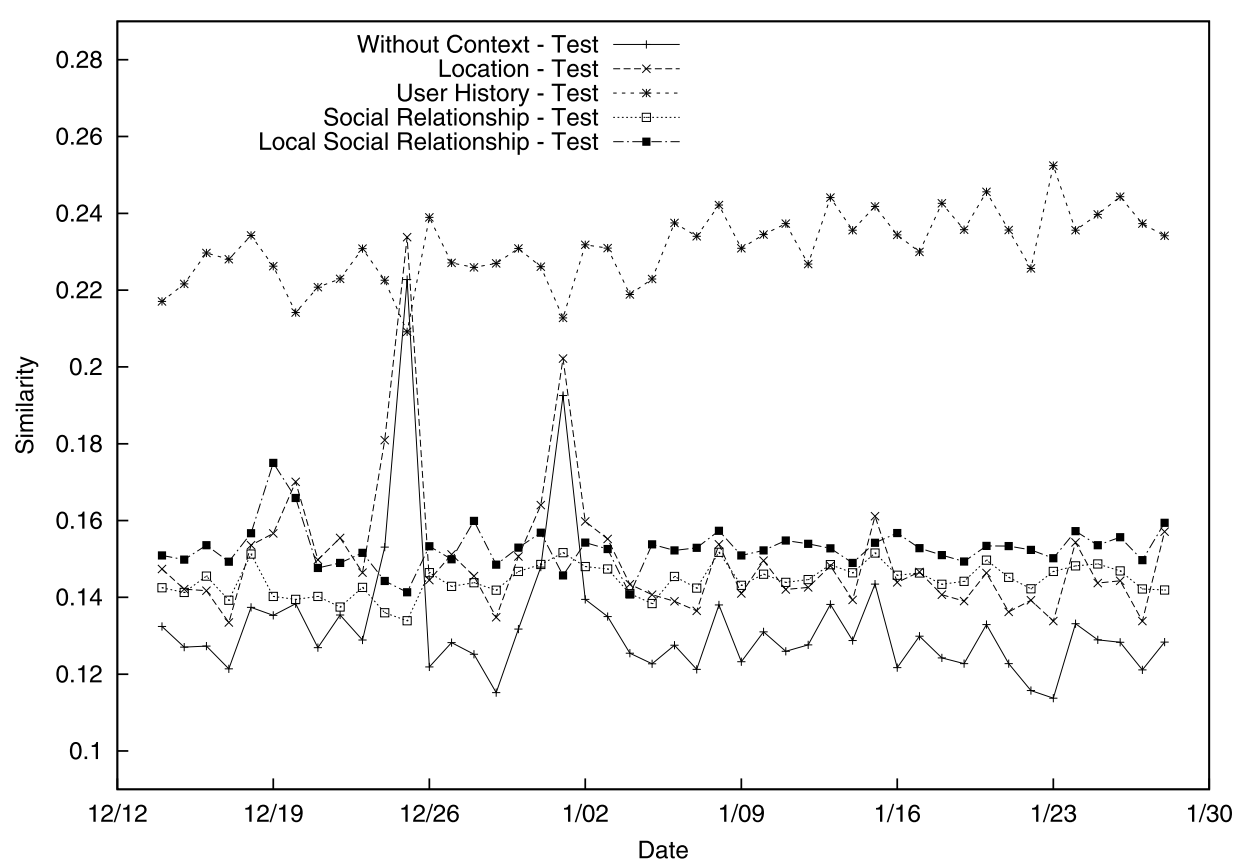

Fig. 2. The analysis result showing the term frequency similarity.

Table 2

Term frequency similarity between two locations

\begin{tabular}{lcccc}
\hline Location & NY & CH & CA & US \\
\hline NY & 0.9866 & 0.8934 & 0.9060 & 0.9044 \\
CH & 0.8909 & 0.9749 & 0.9342 & 0.9184 \\
CA & 0.9060 & 0.9362 & 0.9805 & 0.9444 \\
\hline
\end{tabular}

than the TF similarity of microblogs without context. A remarkable fact of the impact of spatial context is that the impact becomes bigger when local events occur. For instance, from December 19th to 20th, there was a snowstorm in New York. On these days, the difference $(0.0217)$ between the TF similarities of the baseline and the spatial set for NY users is the biggest in our analysis. The snowstorm, a local event, affects microblogs of interest to NY users. We discover that local events, such as weather or games of local sports teams, accelerate the impact of location information.

In addition, we also examine the similarity of TF between sets of microblogs posted in different locations. The approach of this examination is the same. We divide the test set into NY, CH, and CA test sets, according to the user location. The spatial set is also divided into NY, $\mathrm{CH}$, and CA spatial sets. The comparison results are shown in Table 2. Generally, similarity to the same location set is higher than similarity to different locations or the baseline set, US. On December 19th, 2009, the similarity between the two NY sets reached a maximum of 0.992 , but the similarity between NY and other cities was the lowest (0.853). Because of the snowstorm on that day in NY, the frequencies of terms mentioned by users were significantly affected by this spatial context. From this analysis, we can assume that location context is an important factor for finding userpreferred microblog content.

User history is the most influential contextual information in the microblog sets, according to Fig. 2. When the blogging history of a user is used, the similarities depend less on local events or special days compared to other context. The TF similarities of the microblog set collected by user history are always much higher than the baseline TF similarities except on December 25th and January 1st. Their average is 0.231 , the highest similarity among the comparison group. Therefore, there is a strong possibility that users have an interest in topics which they had previously mentioned. For instance, there's a high probability that a user who frequently mentioned terms related to soccer-e.g., 'football' or 'World Cup' - before, will mention the terms again.

The TF similarity of the social microblog set (average: 0.144 ) is slightly higher than that of the baseline set. Because a microblog service is a kind of social networking service, socially contextual information e.g., friendship - also affects it. When users have an interest in an issue, they often exchange opinions about 


\begin{tabular}{|c|c|c|c|c|}
\hline & working & school & shopping & exercising \\
\hline arts & 0.069 & 0.059 & 0.071 & 0.033 \\
\hline business & 0.101 & 0.040 & 0.090 & 0.053 \\
\hline computers & 0.069 & 0.036 & 0.083 & 0.028 \\
\hline games & 0.036 & 0.031 & 0.049 & 0.106 \\
\hline health & 0.079 & 0.059 & 0.038 & 0.152 \\
\hline home & 0.048 & 0.041 & 0.069 & 0.040 \\
\hline news & 0.070 & 0.080 & 0.058 & 0.051 \\
\hline recreation & 0.067 & 0.047 & 0.089 & 0.123 \\
\hline reference & 0.109 & 0.269 & 0.072 & 0.074 \\
\hline science & 0.066 & 0.072 & 0.044 & 0.050 \\
\hline shopping & 0.076 & 0.049 & 0.195 & 0.060 \\
\hline society & 0.161 & 0.133 & 0.069 & 0.057 \\
\hline sports & 0.049 & 0.085 & 0.073 & 0.172 \\
\hline
\end{tabular}

Fig. 3. The distribution of microblogs across categories when terms of an activity are specified.

the issue with on-line friends and forward friends' microblogs which mention the issue using retweet function of microblog services. The similarity results become different if we consider only microblogs posted by a user's friends who are located in the same region as the user. On average, when we consider the local and social microblogs as a set, the TF similarity (0.153) is higher than that of the social set. Also, when there is a local event - e.g., the snowstorm in NY on December 19 th - the similarity $(0.175)$ becomes even higher. According to this observation, we find that there is close dependence between microblogs and social context such as on-line friendship, especially local social networks.

Additionally, we analyze the distribution of microblogs across categories ${ }^{2}$. Figure 3 shows the probabilities of categories when an activity term is mentioned on a microblog. When microblogs contain terms like 'in the school', it is quite probable that the microblogs are related to the reference category, which deals with education, library, and so on. On the other hand, there is a strong likelihood that a microblog is related to the shopping category if it mentions situation terms like 'in the mall' or 'in the shopping center'. Interesting categories are shifted based on terms describing an activity or situation.

From these analysis observations, we can see the impact of contextual information on microblogs. Mi-

\footnotetext{
${ }^{2}$ We refer directories of Open Directory Project (ODP) (http://www.dmoz.org/) for the categories
}

croblogs are affected by context, although this can be to a greater or lesser degree. So, to provide users with interesting microblogs, considering and exploiting context is essential for understanding information needs. Based on these analysis results, in this paper we propose an approach for detecting microblog topics and recommending user-preferred topics.

\section{Context-based microblog topics browser}

In this paper, we design a system which provides mobile users with the microblog topics [11]. We also propose an approach to retrieving and recommending user-preferred microblog topics. Temporal and spatial context are used to detect local microblog topics. We employ user history, social relationships, and activities to select user-preferred topics.

\subsection{Context-aware Mobile Microblog Browser}

We design a system, the Context-aware Mobile Microblog Browser, which provides mobile users with user-preferred microblog topics. Since it is hard to display large quantities of microblogs on a mobile device, we summarize the microblogs as topics that describe recently emerging issues. Microblog topics are displayed on a mobile phone together with microblogs mentioning the topics. It is developed for a mobile phone equipped with sensors, such as a GPS, G-Sensor, and digital compass, which are used to obtain context. It gathers user context from built-in sensors and user profile, detects microblog topics, selects user-preferred topics, and then visualizes them on a mobile device. Figure 1 shows the user interface of the system.

The system consists of a server and a mobile client application. The server detects local microblog topics and selects user-preferred topics. The mobile application gathers context and visualizes the topics detected by the server. The framework of the system is illustrated in Fig. 4. The mobile application deals only with gathering context and visualizing topics; the detection of local microblog topics, the estimating of user interests, and the selection of user-related topics are performed in the server. Context and topics are exchanged between the server and the mobile application.

The server deals with heavy computation tasks with a large amount of microblog data. First, it retrieves local microblogs using location context. Then we com- 


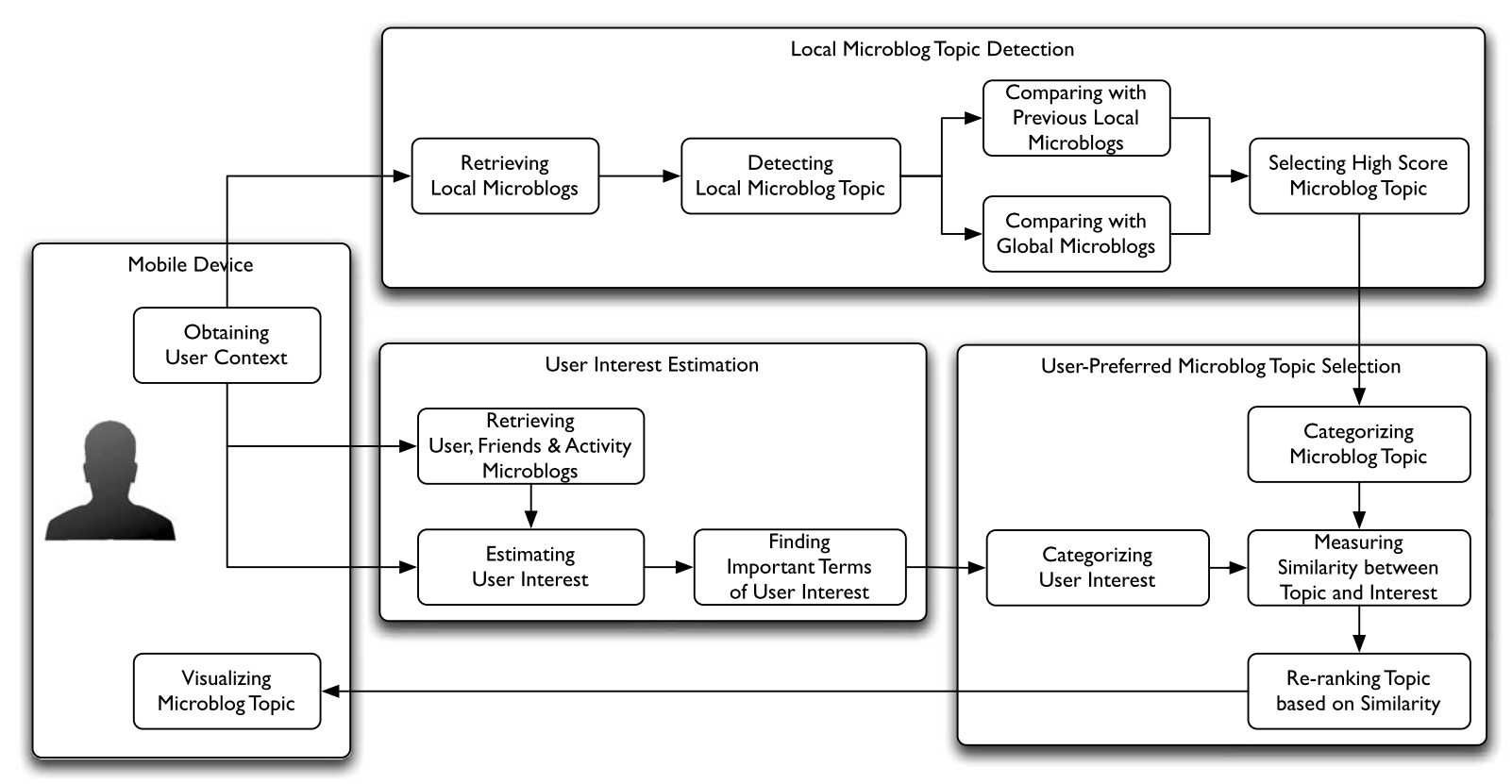

Fig. 4. Framework of the proposed system.

pare them with previous local microblogs and current global microblogs to boost the topic's characteristics of recentness and locality. By way of the comparisons, the server is able to detect local microblog topics. To select user-preferred topics, the server retrieves userrelated microblogs using user history, social relationships, and activities. From the microblogs, the server estimates user interests. To compare detected topics and user interests, it categorizes them into 13 categories used in microblog analysis (see Table 3) and then measures the similarity between topics and interests based on their category vectors. Finally, it re-ranks topics in order of similarity and selects those most related to a user.

The roles of the mobile client application are obtaining user context and visualizing microblog topics detected by the server. Context is gathered from the sensors of a mobile phone. We also take advantage of the user profile, including user history and social relationships. The application transmits the gathered context to the server. It also visualizes topics, together with a map for displaying user location and geotagged microblogs related to the topics, on a mobile phone. Users are able to access recent issues that are frequently mentioned in locations near them. If they want to read further microblogs related to the topics, they can touch the visualized topics and see microblogs containing these topics.
Table 3

Top level categories and their sub-categories in the ODP

\begin{tabular}{ll}
\hline Category & \multicolumn{1}{c}{ Sub-category } \\
\hline arts & movies, television, music, $\ldots$ \\
business & jobs, real estate, investing, $\ldots$ \\
computers & Internet, software, hardware, $\ldots$ \\
games & video games, RPGs, gambling, $\ldots$ \\
health & fitness, medicine, alternative, $\ldots$ \\
home & family, consumers, cooking, $\ldots$ \\
news & media, newspapers, weather \\
recreation & travel, food, outdoors, humor, $\ldots$ \\
reference & maps, education, libraries, $\ldots$ \\
science & biology, psychology, physics, $\ldots$ \\
shopping & clothing, food, gifts, $\ldots$ \\
society & people, religion, issues, $\ldots$ \\
sports & baseball, soccer, basketball, ... \\
\hline
\end{tabular}

\subsection{Local microblog topic detection}

The server in our proposed framework detects local microblog topics for mobile users. In this paper, we define a microblog topic as a term which is frequently mentioned in microblogs. We consider unigram terms only as topics, and consider recently emerging issues instead of steadily mentioned terms - e.g., 'love' and 'day'. For instance, Fig. 5 shows the frequencies of microblog terms 'love' and 'snow' from December 14th, 2009, to December 25th, 2009. The frequencies 


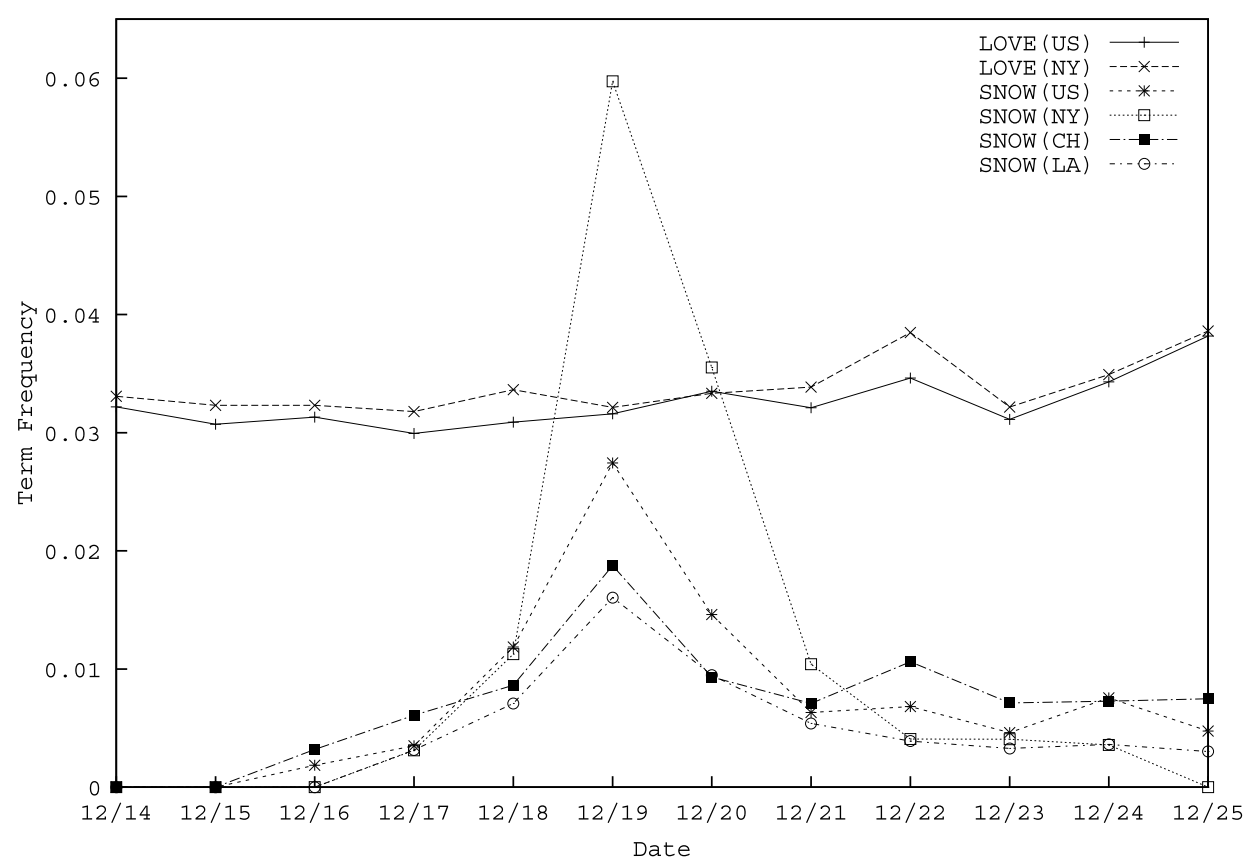

Fig. 5. The microblog frequencies of 'snow' and 'love' in New York, Chicago, California and United States from December 14th, 2009, to December 25th, 2009

of 'love' are even, but the frequencies of 'snow' increase rapidly on December 19th because there was a snowstorm on that day in NY. We do not regard 'love' as a microblog topic, but we do consider 'snow' to be a topic. In addition, 'snow' is considered to be a local microblog topic in NY because its frequency in NY is approximately two times higher than in the rest of the US.

We take advantage of user context, especially spatial and temporal context, to detect local microblog topics. The obtained spatial context - e.g., latitude and longitude from a GPS - is converted into the location and name of a city by reverse-geocoding. We first retrieve microblogs that are posted by users who live in the location. To detect recent rising topics, we exploit temporal context and retrieve previous microblogs. Our approach uses global microblogs retrieved without location context to boost the characteristic of locality. We measure the significance of terms using the context for detecting local microblog topics.

To detect local microblog topics, we measure topic score, which represents the significance of terms. To evaluate the significance of terms so as to detect microblog topics, we define microblog frequency (MF) for a term term as the ratio of the number of microblogs mentioning term to the number of microblogs in a set $\mathbf{B}_{\mathbf{t}}$ in time $t$. The MF is defined as
Eq. (2). Based on computed MF values, we remove terms whose MF value is less than a threshold. We also apply stop-word elimination and a word-stemming process to remove unimportant words, tenses, and plural forms. The higher the MF value of a term, the more frequently the term is mentioned by users. Thus, MF value means the significance of a term for a topic.

$$
\operatorname{MF}\left(\text { term }, \mathbf{B}_{t}\right)=\frac{\mid\left\{b_{t}: \text { term } \in \mathbf{B}_{t}\right\} \mid}{\left|\mathbf{B}_{t}\right|}
$$

We compare MF values with MF values of previous microblog sets and a global microblog set to boost the characteristics of recentness and locality. Terms are compared with frequencies in previous microblog sets to detect recent emerging topics, because we do not treat terms that always have consistent MF values, such as 'love', as a topic. We evaluate the significance of a term based on both the MF value and the inverse of cumulative frequency of the term during certain periods. We propose that Temporal Novelty (TN), the significance of a term term, in time $t$, is defined as

$$
\mathrm{TN}\left(\text { term }, \mathbf{B}_{t}\right)=\frac{\operatorname{MF}\left(\text { term }, \mathbf{B}_{t}\right)}{\frac{1}{n+1} \sum_{i=0}^{n} \operatorname{MF}\left(\text { term }, \mathbf{B}_{(t-\Delta t \cdot i)}\right)}
$$


where $\Delta t$ is a window size and $n$ is the number of previous microblog sets. In addition, terms are compared to the MF value of a microblog set which is retrieved without location context. We define Spatial Novelty (SN) compared with a global microblog set $\mathbf{G B}_{t}$ as Eq. (4) where LB is a set of local microblogs retrieved by location context.

$$
\mathrm{SN}(\text { term })=\frac{\mathrm{MF}\left(\text { term }, \mathbf{L B}_{t}\right)}{\mathrm{MF}\left(\text { term }, \mathbf{G B}_{t}\right)}
$$

The topic score TS, including both temporal and spatial comparison, is estimated by Eq. (5). In the case of Fig. 5, because the topic score of 'snow' on December 19th is larger than that of 'love', 'snow' is considered as a microblog topic in NY. Finally, through temporal-spatial comparison, we select the top $k$ terms as recent local microblog topics based on Eq. (5).

$$
\mathrm{TS}(\text { term })=\mathrm{TN}\left(\text { term }, \mathbf{L B}_{t}\right) \cdot \mathrm{SN}(\text { term })
$$

\subsection{User-preferred microblog topic selection}

After detecting local microblog topics, we apply post-processing to find user-preferred microblog topics. It is a similarity re-ranking approach employing user interests. The proposed approach estimates user interests from user context, including user history, social relationships, and activities. To compare local microblog topics and user interests, we express these in terms of category vectors that represent the probabilities of categories, and compare them in the category vector space. Finally, we re-rank detected local microblog topics based on the similarity to user interests, and select the top $k$ topics.

\subsubsection{User interests estimation}

We employ user interests to select user-preferred microblog topics. We estimate user interests from context, including user history, social relationships, and user activities. We regard microblogs previously posted by a user as user history information, and regard on-line social networks in a microblog service as social relationship information. According to our analysis results in Section 3, both previous microblogs of a user and microblogs of the user's friends reflect interests of the user. Thus, personal information helps us estimate user interests in microblogs. A user's activity is recognized through exploiting context picked up by the sensors of a mobile phone [27]. For instance, GPS positioning can give not only absolute positions but also user activities - e.g., school, working or shopping - because we are able to roughly match a position and an activity. Then, for the sake of matching user context to personal preferences in microblogs, we retrieve microblogs related to the context and find important terms from them.

To find microblog interests, the proposed approach exploits microblogs that are retrieved in accordance with the user context. First, we generate microblog sets related to user history, social relationships, and user activities. Microblogs that were previously posted by a user, are collected for user history. We collect microblogs posted by friends of the user in a microblog service for a microblog set of social relationships. We also collect microblogs that contain terms indicating user activities - for example, 'in the school' or 'in the shopping mall' - for activity context. Then, we find important terms from the collected microblog sets. We assume that frequently mentioned terms are more related to the user's interests than the terms that are mentioned rarely. MF is used to determine the significance of the terms. The MF value means $p($ term $)$, the probability of the occurrence of a term term in a given microblog set. In the case of a user activity microblog set, the MF value means $p$ (term|activity), which represents the probability of a term, given the occurrence of the user activity term activity. The found terms reflect user interests when a user uses a microblog. Through the terms and their MF values, we express a user interest in terms of a category vector indicating the probability of interesting categories.

\subsubsection{Microblog topic and user interests categorization}

To compare a microblog topic and a user interest, we change them into category information. Because it is difficult to measure the similarity between a detected topic and a user interest directly, we consider them to be categories and compare them in category vector space. We define a category vector as the probabilities that a topic or an interest belongs to each category. Each dimension of the vector corresponds to the probability of a separate category. We categorize a topic and an interest according to the significance of terms related to them.

In this paper, we use the categories of the ODP and its descriptions to categorize a topic and an interest. Table 3 shows the categories used in this paper. From ODP's category descriptions, we compute the significance of a term when a category is given. First, we gather descriptions of each category from ODP. Unim- 
portant words of descriptions are removed by stopword elimination. Then we compute the TF-IDF of each term that appears in the descriptions. The TF-IDF values are normalized and used as $p(t \mid c t g)$, a probability of a term $t$ under a category ctg. To obtain the probability of a category when terms are given, we compute $p\left(c t g \mid t_{1}, t_{2}, \ldots, t_{m}\right)$. Using a näive Bayes classifier, it can be estimated by

$$
\begin{aligned}
p\left(c t g \mid t_{1}, t_{2}, \ldots, t_{m}\right) & =\frac{p\left(c t g, t_{1}, t_{2}, \ldots, t_{m}\right)}{p\left(t_{1}, t_{2}, \ldots, t_{m}\right)} \\
& =\frac{p(c t g) \prod_{j=1}^{m} p\left(t_{j} \mid c t g\right)}{p\left(t_{1}, t_{2}, \ldots, t_{m}\right)} \\
& \propto p(c t g) \prod_{j=1}^{m} p\left(t_{j} \mid c t g\right)
\end{aligned}
$$

because the occurrences of terms are independent and the denominator $p\left(t_{1}, t_{2}, \ldots, t_{m}\right)$ does not depend on a category $c t g$. A näive Bayes classifier is robust to noise terms because such terms are averaged out when estimating conditional probabilities. Also, it is robust to irrelevant terms $t_{x}$, because $p\left(t_{x} \mid c t g\right)$ becomes almost uniformly distributed and has no impact on the overall computation of the posterior probability. Due to these characteristics of term occurrences, we use a näive Bayes classifier to estimate probabilities that a topic or an interest belongs to each category.

We categorize a topic and an interest as categories according to terms that appeared with the topic and the interest in microblogs. The category vector ctg for a topic or an interest is $\mathbf{c t g}=\left[c_{1}, c_{2}, \ldots, c_{n}\right]^{T}$ where $c_{i}$ is the probability that the topic or the interest belongs to the category $\mathrm{ctg}$,

$$
c_{i}=p\left(c t g_{i}\right) \prod_{j=1}^{m} p\left(t_{j} \mid c t g_{i}\right) \cdot w\left(t_{j}\right)
$$

$n$ is the number of categories, and $t_{j}$ is a term that appeared together with the topic or the interest in microblogs. For example, a topic 'snow' can be represented as $[0.054,0.032, \ldots, 0.108]$, which means that the probability of the arts category is 0.054 , and the probability of the sports category is 0.108 . We give weight $w\left(t_{j}\right)$ to the significance of a term $t_{j}$. It is estimated by the MF of microblogs that mention a topic or an interest for categorization. After normalization of $c_{i}$, we obtain a category vector ctg for a topic or an interest.

\subsubsection{Microblog topic re-ranking}

We re-rank detected local microblog topics according to the similarity between the topics and user interests. Our approach to re-ranking topics is to compute each topic's similarity to the estimated user interests, and to re-rank the topics according to the similarity scores. We build category vectors for both a topic and an interest by Eq. (7). Then we compute the normalized Cosine similarity score between the topic and the interest expressed as category vectors. The similarity is estimated by

$$
\operatorname{Ctg} \operatorname{Sim}(\mathbf{t p}, \mathbf{i n})=\frac{\sum_{i=1}^{n} c_{i, \mathbf{t p}} \cdot c_{i, \mathbf{i n}}}{\sqrt{\sum_{i=1}^{n} c_{i, \mathbf{t} \mathbf{p}}^{2}} \cdot \sqrt{\sum_{i=1}^{n} c_{i, \mathbf{i n}}^{2}}}
$$

for a topic, tp, and an interest, in. Finally, the topics are re-ranked in order of the values that are computed by multiplying the similarity by the topic score Eq. (5). The re-ranking score for a topic tp and an interest in is estimated by Eq. (9). We select the top $k$ microblog topics and provide users with them.

$$
\mathrm{RS}(\mathbf{t p}, \mathbf{i n})=\mathrm{TS}(\mathbf{t p}) \cdot \operatorname{CtgSim}(\mathbf{t p}, \mathbf{i n})
$$

\section{Evaluation}

In this paper, we proposed an approach to detecting microblog topics and selecting user-preferred topics based on mobile user context. To verify the suitability of the proposed approach, we evaluated its effectiveness based on its relevance to a user of retrieved microblog topics. We also evaluated the order of ranked microblog topics. To evaluate the impact of context, we compared the results of our approach with microblog topics retrieved without context on the same microblog data sets. To evaluate the impact of post-processing, we also compared the results with microblog topics retrieved with spatial context.

In this evaluation, we took advantage of microblog data sets that are used in the analysis of the relationship between microblog and context, as described in Section 3. We used Twitter as a sample microblog service. Microblogs posted by US users are regarded as a global data set, and microblogs posted by NY users are regarded as a local data set. We used user activities as described in Fig. 3. In addition, we used users' following information as social relationships in Twitter. In order to remove rare terms, we set a $0.5 \%$ threshold value for MF. 


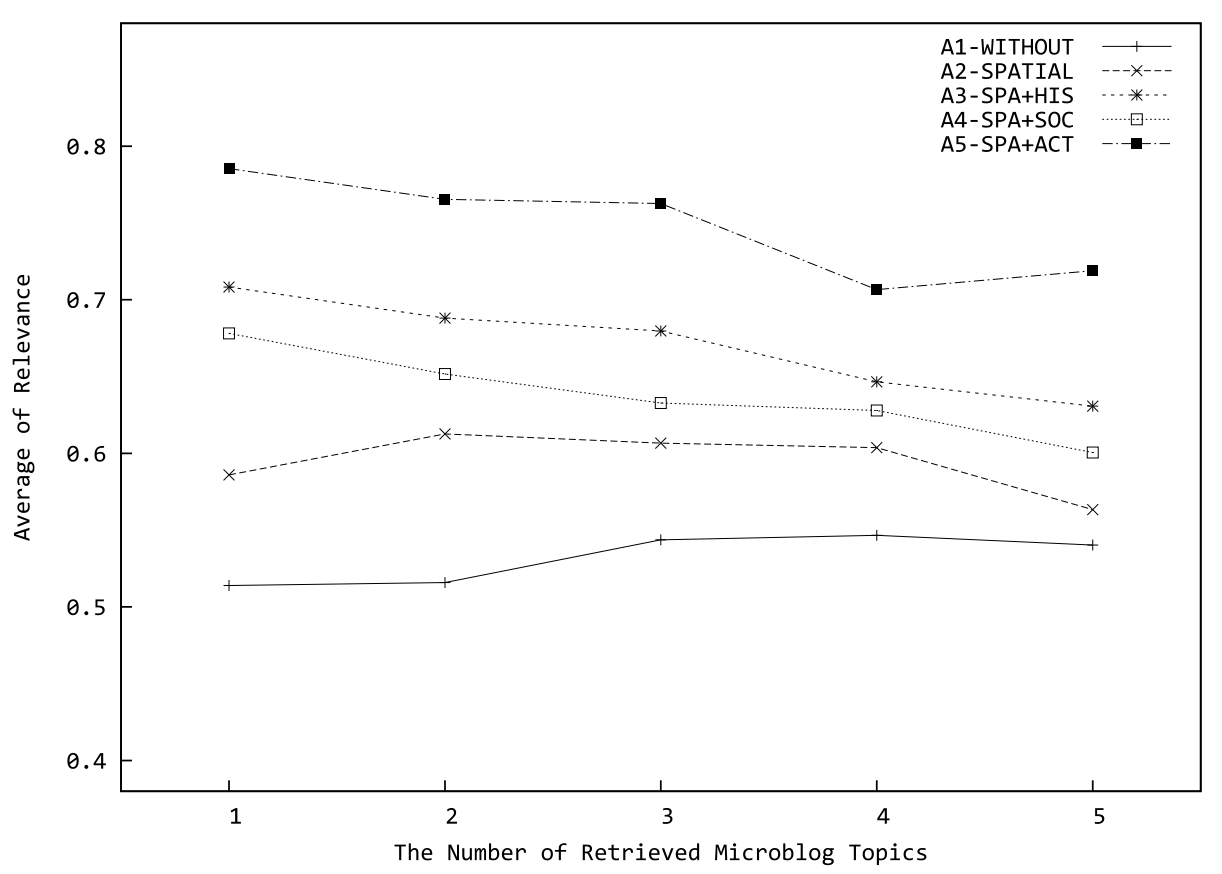

Fig. 6. The evaluation results showing the average of the topics' relevance by different algorithms and the number of retrieved microblog topics.

\subsection{Evaluation approach}

Evaluation framework We divided the microblog data set into two sets. Randomly selected $75 \%$ of the microblogs were used as a training set and the others were used as a test set. The training set was used to generate microblog topics. We compared the generated topics with each microblog of the test set. We defined a microblog topic's relevance score, which represents similarity between a microblog, $b$, and a microblog topic, $t p$, as:

$$
\operatorname{rel}(b, t p)= \begin{cases}1 & \text { if } t p \in b \\ \operatorname{CtgSim}\left(\operatorname{ctg}_{b}, \mathbf{c t g}_{t p}\right) & \text { otherwise }\end{cases}
$$

i.e., the score is 1 if a microblog contains a microblog topic, or the score is computed by the similarity between the topic and the microblog in category vector space.

In addition, we evaluated the order of selected microblog topics using the nDCG [12]. We recruited 5 participants who experienced microblog services. They evaluated the results of topic detection and selection. We provided them with microblog topics and microblogs of the test set including not only topics selected by our approach, but also topics selected by other approaches that detect microblog topics without employing context or without selecting user-preferred microblog topics. The participants evaluated the relevance between topics and each microblog. Finally, we used nDCG to compute the suitability of topics' ranking according to the evaluated scores.

Ground truth We regarded terms mentioned in the microblogs of the test set as the ground truth to evaluate the retrieved microblog topics. Because a microblog posted by a user reflects the user's interests, we used Eq. (10) to compute the relevance of a topic. If a microblog contains a topic, we assumed that the topic was correctly selected for the user. If a microblog did not contain a topic, we used a category vector of the microblog as the ground truth. For the ground truth of the nDCG, the topics, listed in descending order in accordance with evaluated scores by participants, were regarded as ideal topics for a user.

Baseline approach We compared our proposed approach with other approaches that detect microblog topics without context or with only spatial context. As a base approach, we used only Eq. (3) to detect global microblog topics. To see the impact of our postprocessing that selects user-preferred topics based on user interests, we also used an approach that detects local microblog topics as a baseline approach. These approaches were compared with our approach to determine the impact of spatial context and our postprocessing method. 


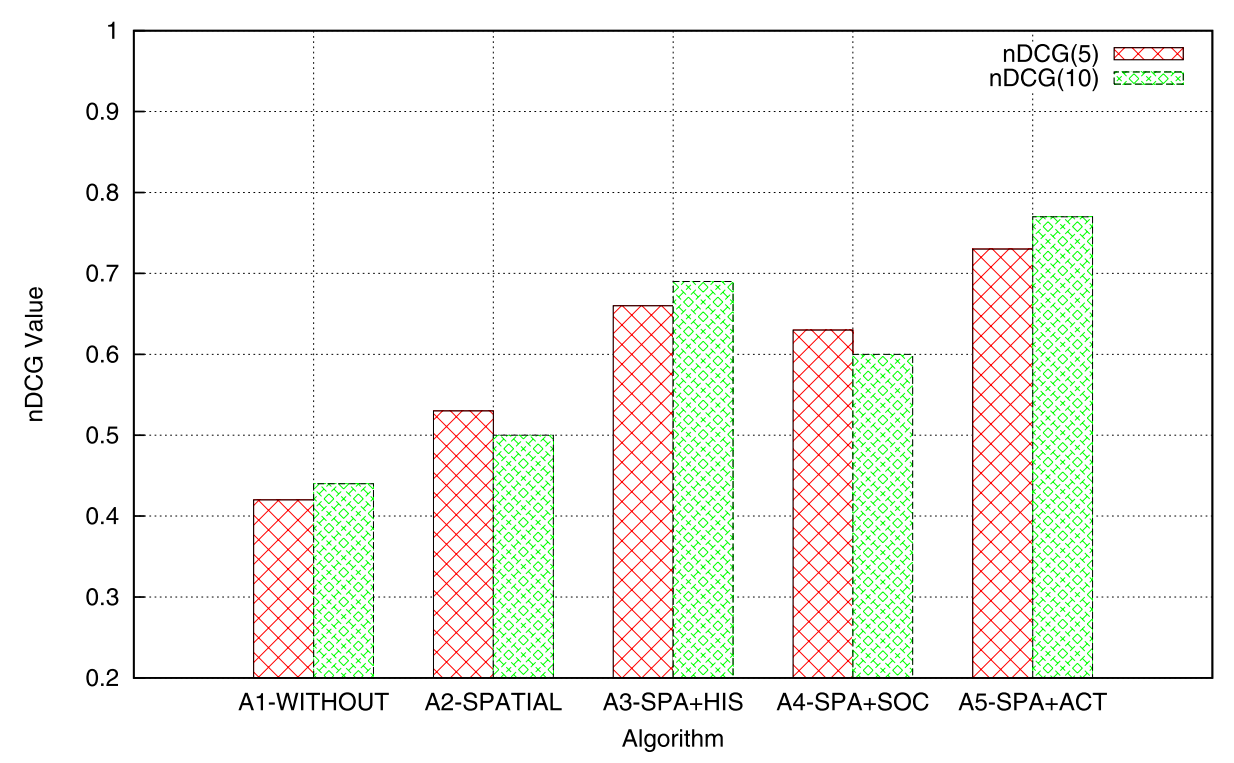

Fig. 7. The evaluation results showing the $\mathrm{nDCG}$ values for comparing different algorithms when we retrieve 5 topics and 10 topics.

\subsection{Evaluation results}

The results of the evaluation that computes the relevance of detected topics and the microblogs in a test set are illustrated in Fig. 6. The x-axis of Fig. 6 is the number of retrieved topics, and the $\mathrm{y}$-axis is the average of the topics' relevance based on Eq. (10). As Fig. 6 shows, the relevance of retrieved microblog topics to microblogs of the test set is higher when we take advantage of user context to detect microblog topics. When we detect microblog topics using Eq. (3) without using context as the baseline approach, the relevance is the lowest (average: 0.531). On the other hand, when we take advantage of spatial context to detect local microblog topics, the relevance of local topics is relatively high in comparison with the baseline approach (the average of local topics' relevance is 0.592). However, when 5 microblog topics are retrieved, the gap between the relevance of global topics and that of local topics is less than 0.02 . The reason for the small gap is that we compute the average relevance of topics regardless of their rank in this evaluation.

We also determine that the relevance of retrieved microblog topics is enhanced when we take advantage of post-processing based on user interests. Overall, the relevance of topics with post-processing is higher than those without post-processing, as shown in Fig. 6. Microblog topics using post-processing with user activity (average: 0.748 ) are higher than topics using any other approaches. Employing user history and user social relationships also helps to improve the relevance of retrieved topics. Therefore, our approach, which estimates user interests and re-ranks topics based on the interest, enhances microblog topic retrieval. In postprocessing approaches, the relevance generally decreases if the number of retrieved topics increases. The reason for the decrease is also related to the ranking of topics.

To see the impact of the order of retrieved topics, we evaluated topics' relevance based on the nDCG measurement. Figure 7 shows the results of the nDCG evaluation using different algorithms and the number of retrieved topics. Like in Fig. 6, when microblog topics are detected without context, the nDCG value is lower than the other algorithms in this evaluation. The $\mathrm{nDCG}$ values of algorithms with post-processing exceed 0.6 whether we use the first 5 retrieved topics or 10 topics. They are higher than A1 (without context) and A2 (with spatial context) by 0.24 and 0.13 , respectively. The most effective algorithm is A5, which uses spatial context to detect local topics and user activities to select user-preferred topics. Compared to Fig. 6, the gaps between the nDCG values of global topics and local topics are bigger whether we retrieve 5 microblog topics or 10 topics. Also, although the nDCG values of A 2 and A4 (with spatial and social context) are slightly decreased when we retrieve 10 topics, the number of retrieved topics does not significantly affect the relevance. In Fig. 7, we can see that our context-based ap- 
proach improves the relevance of retrieved topics with their ranks.

Here, we give examples to illustrate the effectiveness of our approach. On December 19, the majority of retrieved NY microblog topics were related to snow, such as 'snow', 'snowstorm', and 'weather'. However, the retrieved topics without spatial context were '\#followfriday', 'Avatar', and 'snow'. On that day, the relevance of the local topics was much higher than that of the global topics. On December 25, topics from the global and local microblogs were almost the same: 'Christmas', 'Jesus', and '\#celebgift'. In this case, the gap of the relevance between global and local topics was very small. However, when post-processing was used, the relevance was improved. The system moved '\#celebgift' to the first rank when a user went shopping. For a user who usually mentioned terms related to sports, our approach selected a topic, 'Kobe', which is the name of a famous basketball player who played a game on that day. From these examples, we can see how the relevance was improved based on different algorithms.

\subsection{Discussion}

Because microblog usage is affected by user context, as we observed in Section 3.3, mobile user context is an important factor to retrieve user-preferred microblog content. As shown in the experimental results, our approach employing user context helps mobile information retrieval systems understand information needs of mobile users. Temporal context helps our system detect timely issues, and spatial context helps the system narrow down the search range of microblogs. The other context improves the selection of content based on user interests.

In the results, the approaches with post-processing - i.e., A3, A4 and A5 - enhance the relevance of retrieved topics much more than A2. It clearly shows that re-ranking topics by similarity to the user interests gives a large improvement in the relevance. Furthermore, user activity information is the most effective context to enhance the selection of user-preferred content. It would be interesting future work to refine and improve upon the user interests estimation technique presented in this paper.

However, there are number of limitations in our approach. Since it is hard to compute the similarity between a microblog topic and a user interest directly, our approach converts them into category vectors. The category vector is based on the 13 main categories of the ODP. Because of the specificity of the categories, some topics are incorrectly selected. For instance, a user has an interest in baseball, but our approach provides the user with topics related to basketball. The reason why the basketball topics are provided with is that we treat the user interest as sports. To avoid this problem, we need to consider fine-grained categories. In addition, it is difficult to make a user interest estimation model that reflects priorities of context. That is the reason why we combine spatial context with only one of the other context to post-process retrieved topics. We need to consider an approach that combines the context dynamically.

When a user browses information on a mobile device, mobile information visualization is also important as well as mobile information retrieval. It is necessary to consider content adaptation for small display of a mobile device. We summarized microblogs as topics describing recently emerging local issues, instead of visualizing all microblogs of a user's friends. However, in our evaluation, we concentrated on the relevance of retrieved topics. To verify the effectiveness of topics for browsing information on a mobile device, we need to consider a qualitative analysis that compares microblog topic visualization with traditional microblog visualization. Our current approach can be improved by considering these issues.

\section{Conclusion and future work}

In this paper, we studied the problem of finding content relevant to the information needs of mobile users. We presented the findings of a large-scale study on the impact of user context on microblog usage, and introduced a system that provides mobile users with user-preferred microblog topics. From our observation, we discovered that user context, such as time, locations, user history, and social relationships, affects the term frequencies of microblogs. We also discovered that interesting categories are shifted based on user activities. Using these observation results, we were able to develop an approach that finds userpreferred microblog content for mobile users. Our evaluation showed that the proposed approach enhanced the relevance of microblog topics to information needs of mobile users. We expect that our proposed approach can effectively enhance information retrieval on a mobile device.

However, there is still some future work necessary to improve the relevance of the retrieved microblog 
topics. In this work, only spatial context was combined with user history, social relationships, and user activities. We need to consider an approach that combines these context dynamically or finds the most effective factor for each individual user. Although our approach concentrates on only user context, environmental context also can be used to help mobile applications understand information needs of mobile users. We will continue to investigate the possibilities of the environmental context for reducing the burden of mobile information retrieval. We expect that our proposed approach can be extended for retrieving timely social media or information search.

\section{Acknowledgements}

This research was supported by Microsoft Research Asia. It was done when Jonghyun Han, the author of this paper, was an intern at Microsoft Research Asia.

\section{References}

[1] G.D. Abowd, C.G. Atkeson, J. Hong, S. Long, R. Kooper, and M. Pinkerton, Cyberguide: A mobile context-aware tour guide, Wirel. Netw. 3(5) (1997), 421-433.

[2] R.A. Baeza-Yates and B. Ribeiro-Neto, Modern Information Retrieval, Addison-Wesley Longman Publishing Co., Inc., Boston, MA, USA, 1999.

[3] T. Brants, F. Chen, and A. Farahat, A system for new event detection, in: Proc. of the 26th Annual International ACM SIGIR Conference on Research and Development in Informaion Retrieval, SIGIR '03, ACM, New York, NY, USA, 2003, pp. 330-337.

[4] P.J. Brown and G.J.F. Jones, Context-aware retrieval: Exploring a new environment for information retrieval and information filtering, Personal Ubiquitous Comput. 5(4) (2001), 253 263.

[5] J. Chen, R. Nairn, and E. Chi, Speak little and well: Recommending conversations in online social streams, in: Proc. of the 2011 Annual Conference on Human Factors in Computing Systems, CHI' 11, ACM, New York, NY, USA, 2011, pp. 217226.

[6] K.-Y. Chen, L. Luesukprasert, and S.-c.T. Chou, Hot topic extraction based on timeline analysis and multidimensional sentence modeling, IEEE Trans. on Knowl. and Data Eng. 19(8) (2007), 1016-1025.

[7] K. Church, J. Neumann, M. Cherubini, and N. Oliver, Socialsearchbrowser: A novel mobile search and information discovery tool, in: Proc. of the 15th International Conference on Intelligent User Interfaces, IUI '10, ACM, New York, NY, USA, 2010, pp. 101-110.

[8] K. Church and B. Smyth, Who, what, where \& when: A new approach to mobile search, in: Proc. of the 13th International Conference on Intelligent User Interfaces, IUI '08, ACM, New York, NY, USA, 2008, pp. 309-312.
[9] K. Church and B. Smyth, Understanding the intent behind mobile information needs, in: Proc. of the 14th International Conference on Intelligent User Interfaces, IUI '09, ACM, New York, NY, USA, 2009, pp. 247-256.

[10] P. Coppola, V. Della Mea, L. Di Gaspero, D. Menegon, D. Mischis, S. Mizzaro, I. Scagnetto, and L. Vassena, The contextaware browser, Intelligent Systems, IEEE 25(1) (2010), 38-47.

[11] J. Han, X. Xie, and W. Woo, Context-based local hot topic detection for mobile user, in: Adjunct Proc. of the 8th International Conference on Pervasive Computing, Springer, 2010, pp. 5-8.

[12] K. Järvelin and J. Kekäläinen, Cumulated gain-based evaluation of ir techniques, ACM Trans. Inf. Syst. 20 (2002), 422446.

[13] M. Kamvar and S. Baluja, A large scale study of wireless search behavior: Google mobile search, in: Proc. of the SIGCHI Conference on Human Factors in Computing Systems, CHI '06, ACM, New York, NY, USA, 2006, pp. 701-709.

[14] S. Lemmelä, A. Vetek, K. Mäkelä, and D. Trendafilov, Designing and evaluating multimodal interaction for mobile contexts, in: Proc. of the 10th International Conference on Multimodal Interfaces, ICMI '08, ACM, New York, NY, USA, 2008, pp. 265-272.

[15] A. Marcus, M.S. Bernstein, O. Badar, D.R. Karger, S. Madden, and R.C. Miller, Twitinfo: Aggregating and visualizing microblogs for event exploration, in: Proc. of the $2011 \mathrm{An}$ nual Conference on Human Factors in Computing Systems, CHI '11, ACM, New York, NY, USA, 2011, pp. 227-236.

[16] S. Morinaga and K. Yamanishi, Tracking dynamics of topic trends using a finite mixture model, in: Proc. of the Tenth ACM SIGKDD International Conference on Knowledge Discovery and Data Mining, KDD '04, ACM, New York, NY, USA, 2004, pp. 811-816.

[17] T. Sakaki, M. Okazaki, and Y. Matsuo, Earthquake shakes twitter users: Real-time event detection by social sensors, in: Proc. of the 19th International Conference on World Wide Web, WWW'10, ACM, New York, NY, USA, 2010, pp. 851-860.

[18] Y. Sekiguchi, H. Kawashima, H. Okuda, and M. Oku, Topic detection from blog documents using users' interests, in: Proc. of the 7th International Conference on Mobile Data Management, MDM '06, IEEE Computer Society, Washington, DC, USA, 2006, pp. 108-111.

[19] K. Sparck Jones, A statistical interpretation of term specificity and its application in retrieval, in: Document Retrieval Systems, Taylor Graham Publishing, London, UK, 1988, pp. 132-142.

[20] J.-H. Su, H.-H. Yeh, P. Yu, and V. Tseng, Music recommendation using content and context information mining, Intelligent Systems, IEEE 25(1) (2010), 16-26.

[21] X. Sun and A. May, The role of spatial contextual factors in mobile personalization at large sports events, Personal Ubiquitous Comput. 13(4) (2009), 293-302.

[22] S. Tamminen, A. Oulasvirta, K. Toiskallio, and A. Kankainen, Understanding mobile contexts, Personal Ubiquitous Comput. 8(2) (2004), 135-143.

[23] F.S. Tsai, M. Etoh, X. Xie, W.-C. Lee, and Q. Yang, Introduction to mobile information retrieval, Intelligent Systems, IEEE 25(1) (2010), 11-15.

[24] Z. Xu and Y. Yuan, The impact of context and incentives on mobile service adoption, Int. J. Mob. Commun. 7(3) (2009), 363-381. 
[25] Y. Yang, J. Zhang, J. Carbonell, and C. Jin, Topic-conditioned novelty detection, in: Proc. of the Eighth ACM SIGKDD International Conference on Knowledge Discovery and Data Mining, KDD '02, ACM, New York, NY, USA, 2002, pp. 688-693.

[26] J. Yi, F. Maghoul, and J. Pedersen, Deciphering mobile search patterns: A study of yahoo! mobile search queries, in: Proc. of the 17th International Conference on World Wide Web, WWW '08, ACM, New York, NY, USA, 2008, pp. 257-266.

[27] Y. Zheng, Q. Li, Y. Chen, X. Xie, and W.-Y. Ma, Understanding mobility based on gps data, in: Proc. of the 10th International Conference on Ubiquitous Computing, UbiComp '08, ACM, New York, NY, USA, 2008, pp. 312-321. 